\title{
Expression of specific chemokines and chemokine receptors in the central nervous system of multiple sclerosis patients
}

\author{
Torben L. Sørensen, ${ }^{1,2}$ Marie Tani, ${ }^{1}$ Jakob Jensen, ${ }^{2}$ Virginia Pierce,${ }^{1}$ Claudia Lucchinetti, ${ }^{3}$ \\ Virginia A. Folcik, ${ }^{1}$ Shixin Qin, ${ }^{4}$ Jim Rottman, ${ }^{4}$ Finn Sellebjerg, ${ }^{2}$ Robert M. Strieter, ${ }^{5}$ \\ Jette L. Frederiksen, ${ }^{2}$ and Richard M. Ransohoff ${ }^{1}$ \\ ${ }^{1}$ Department of Neurosciences, The Lerner Research Institute and Mellen Center for Multiple Sclerosis Treatment and Research, \\ Cleveland Clinic Foundation, Cleveland, Ohio 44195, USA \\ 2Department of Neurology, Glostrup Hospital, University of Copenhagen, DK-2600 Glostrup, Denmark \\ ${ }^{3}$ Department of Neurology, Mayo Clinic, Rochester, Minnesota 55905, USA \\ ${ }^{4}$ LeukoSite Inc., Cambridge, Massachusetts 02142, USA \\ ${ }^{5}$ Department of Internal Medicine, The University of Michigan Medical Center, Ann Arbor, Michigan 48109-0638, USA
}

Address correspondence to: Richard M. Ransohoff, Department of Neurosciences, NC30, The Lerner Research Institute, Cleveland Clinic Foundation, 9500 Euclid Avenue, Cleveland, Ohio 44195, USA. Phone: (216) 444-0627; Fax: (216) 444-7927;

E-mail: ransohr@cesmtp.ccf.org

Torben L. Sørenson and Marie Tani contributed equally to this work.

Received for publication September 8, 1998, and accepted in revised form January 27, 1999.

\begin{abstract}
Chemokines direct tissue invasion by specific leukocyte populations. Thus, chemokines may play a role in multiple sclerosis (MS), an idiopathic disorder in which the central nervous system (CNS) inflammatory reaction is largely restricted to mononuclear phagocytes and $\mathrm{T}$ cells. We asked whether specific chemokines were expressed in the CNS during acute demyelinating events by analyzing cerebrospinal fluid (CSF), whose composition reflects the CNS extracellular space. During MS attacks, we found elevated CSF levels of three chemokines that act toward T cells and mononuclear phagocytes: interferon- $\gamma$-inducible protein of $10 \mathrm{kDa}$ (IP-10); monokine induced by interferon- $\gamma$ (Mig); and regulated on activation, normal T-cell expressed and secreted (RANTES). We then investigated whether specific chemokine receptors were expressed by infiltrating cells in demyelinating MS brain lesions and in CSF. CXCR3, an IP-10/Mig receptor, was expressed on lymphocytic cells in virtually every perivascular inflammatory infiltrate in active MS lesions. CCR5, a RANTES receptor, was detected on lymphocytic cells, macrophages, and microglia in actively demyelinating MS brain lesions. Compared with circulating T cells, CSF T cells were significantly enriched for cells expressing CXCR3 or CCR5. Our results imply pathogenic roles for specific chemokine-chemokine receptor interactions in MS and suggest new molecular targets for therapeutic intervention.
\end{abstract}

J. Clin. Invest. 103:807-815 (1999).

\section{Introduction}

Multiple sclerosis (MS), an inflammatory, demyelinating disorder of the human central nervous system (CNS), is the leading cause of nontraumatic neurological disability among young adults in North America (1). Progressive neurological impairment frequently develops during the course of the disease, probably because of irreversible tissue injury (1). Current MS treatments include interferons, corticosteroids, and cytotoxic immunosuppressive agents, often with unsatisfactory outcomes (2). The etiology of the disease remains uncertain but is widely considered to involve organ-specific autoimmune destruction of CNS myelin (3). Innovative therapies for MS seek to abrogate this specific autoreactivity (4).

The histopathology of the MS lesion is characterized by infiltration of the CNS by inflammatory leukocytes, which are considered critical for disease pathogenesis. Inflammatory CNS events during the course of MS are detected in vivo by magnetic resonance imaging or by virtue of elevated leukocyte counts in the cerebrospinal fluid (CSF). Recent reports documented a direct correlation between CNS inflammation and clinical progression of MS; these findings focused attention on devel- oping MS treatment strategies to inhibit leukocyte invasion of the CNS $(2,4,5)$. For example, novel agents that specifically target membrane glycoproteins associated with leukocyte extravasation are under investigation in MS clinical trials $(6,7)$.

Investigators seeking to regulate inflammation are guided by extensive research into the molecular mechanisms of leukocyte extravasation (8-10). The blood-brain barrier (BBB), which excludes circulating macromolecules and cells from the CNS, poses an additional consideration for the design of MS therapeutics. Two categories of molecules direct leukocyte migration into inflammatory sites: adhesion molecules and chemoattractants. Among chemoattractants, chemokines (small, proinflammatory chemotactic cytokines) have attracted particular interest because of their potential role in pathogenic inflammation (11-13). Chemokines selectively attract leukocyte subsets; some chemokines act specifically toward neutrophils or eosinophils, others toward monocytes, dendritic cells, or T cells (12). Chemokines appear to act in at least two ways: first, through direct chemoattraction, and second, by activating leukocyte integrins to bind their adhesion receptors on endothelial cells $(13,14)$. Because 
Table 1

CSF chemokine analysis: patient characteristics and CSF formula

\begin{tabular}{|c|c|c|c|c|c|c|}
\hline Diagnosis & Number & $r$ Gender & $\begin{array}{c}\text { Age } \pm \text { SD } \\
\text { (years) }\end{array}$ & $\begin{array}{c}\text { Mean CSF WBC: } \\
\text { cells } / \mu \mathrm{l} \pm \mathrm{SD} \\
(\text { range })\end{array}$ & $\begin{array}{c}\text { Abnormal CSF } \\
\text { oligoclonal bands } \\
(\%)\end{array}$ & $\begin{array}{c}\text { Abnormal CSF/serum } \\
\text { albumin ratio }{ }^{\mathrm{A}} \\
(\%)\end{array}$ \\
\hline$M S^{B}$ & 382 & $21 \mathrm{~F} / 17 \mathrm{M}$ & $34 \pm 9$ & $13 \pm 10(2-33)$ & 100 & 2.6 \\
\hline $\mathrm{CON}^{\mathrm{C}}$ & 21 & $12 \mathrm{~F} / 9 \mathrm{M}$ & $48 \pm 15$ & $2 \pm 1(0-4)$ & 0 & 0 \\
\hline ONDD & 10 & $4 \mathrm{~F} / 6 \mathrm{M}$ & $59 \pm 15$ & $2 \pm 1(1-3)$ & 10 & 20 \\
\hline
\end{tabular}

Alndicates disruption of blood-brain barrier; see Methods for calculations. ${ }^{B}$ All had recent symptoms of active demyelinating disease: optic neuritis with or without other symptoms $(n=28)$; recurrence of MS symptoms $(n=5)$; isolated brainstem or spinal cord attack $(n=5)$. CHerniated lumbar disc $(n=9)$ or mechanical low back pain $(n=7)$; headache with meningitis ruled out $(n=4)$; brachial plexopathy $(n=1)$. ${ }^{C}$ Cervical spondylotic myelopathy $(n=5)$; cerebral metastasis $(n=1)$; carcinomatous meningitis $(n=1)$; stroke, ALS, axonal polyradiculoneuropathy ( $n=1$ each). CSF, cerebrospinal fluid; CON, non-neurological control; $F$, female; $M$, male; MS, multiple sclerosis; $O N D$, other neurological diseases.

of their number and diversity, chemokines present a bewildering complexity; only recently have specific functions of chemokines in physiology and disease begun to be elucidated. Much of this progress has been made through construction of transgenic and knockout mice, permitting analysis of chemokine action in vivo (15). Strikingly, studies using transgenic mice that overexpressed chemokines under control of tissue-specific promoters indicated that chemokines are sufficient to direct migration of target leukocytes into the CNS (16-18).

Several research groups have used experimental autoimmune encephalomyelitis (EAE), a model for MS, to probe CNS chemokine expression and function (19, $20)$. Karpus and colleagues $(21,22)$ showed that anti-macrophage inflammatory protein- $1 \alpha$ (MIP- $1 \alpha)$ antibodies suppressed initial attacks of adoptive-transfer EAE, while anti-monocyte chemotactic protein-1 (MCP-1) antibodies inhibited subsequent relapses, indicating that specific chemokines execute nonredundant functions in this disease model. These findings lent functional significance to prior analyses showing vigorous CNS chemokine expression during EAE, with a remarkably consistent relationship to clinical disease activity (21-30). Importantly, the temporal and spatial patterns of chemokine expression correlated tightly with the distribution of CNS inflammatory infiltrates in EAE (20). Chemokines also influence leukocyte infiltration into the CNS during posttraumatic and ischemic responses $(23,31-36)$.

Chemokines have been documented in acute and chronic human inflammatory disorders (11). Therefore, results from both experimental models and clinical material motivated consideration of chemokine expression in MS. Examination of MS autopsy material confirmed the distribution of chemokines initially delineated in chronic relapsing murine EAE: MCP-1 was expressed principally by astrocytes near inflammatory infiltrates, while MIP- $1 \alpha$ and regulated on activation, normal T-cell expressed and secreted (RANTES) were produced by perivascular inflammatory cells $(25,28,37-39)$. MIP-1 $\alpha$ was detected at low levels in the CSF of patients with MS and other inflammatory disorders, but not in samples from healthy control subjects (40). CSF levels of other chemokines in MS have not been reported.

In this report, we describe studies of chemokines and chemokine receptors in the CNS in active MS. We studied members of the two major chemokine subgroups: the CXC family, in which two conserved cysteines are separated by a single residue, and the $\mathrm{CC}$ chemokines, in which the two cysteines are adjacent. Elevated concentrations of two CXC chemokines, interferon- $\boldsymbol{\gamma}$-inducible protein of $10 \mathrm{kDa}$ (IP-10) and monokine induced by interferon- $\gamma$ (Mig), were detected in the CSF of patients with symptomatic attacks of inflammatory demyelination. CSF levels of the CC chemokine RANTES were also elevated in MS patients. Moreover, $\mathrm{CD}^{+} \mathrm{T}$ cells that expressed the type 3 CXC chemokine receptor (CXCR3), an IP-10/Mig receptor, were enriched in the CSF compared with the systemic circulation. $\mathrm{CD}^{+}$and $\mathrm{CD}^{+} \mathrm{T}$ cells in the CSF also expressed type 5 CC chemokine receptor (CCR5), a RANTES receptor, much more frequently than in peripheral blood. Immunohistochemical studies of inflammatory MS brain lesions demonstrated that CXCR3-positive mononuclear cells were an abundant and invariant component of the CNS leukocyte infiltrate. Actively demyelinating MS lesions also contained large numbers of CCR5positive lymphocytic cells, macrophages, and microglia. CCR1 and CCR3, alternative RANTES receptors, were detected less frequently. Taken in their entirety, these data imply specific roles for IP-10, Mig, and CXCR3, and for RANTES and CCR5, in the MS inflammatory process. Thus, these results suggest new molecular targets for therapeutic intervention in MS.

\section{Methods}

Patients. The study was approved by the Scientific Ethics Committee of the Government of Denmark and by the Institutional Review Board of the Cleveland Clinic Foundation. Where mandated, written consent was obtained from all participants.

Two groups of patients were studied. From the first group $(n=69$; Table 1), CSF was obtained by lumbar puncture (LP) for chemokine analysis by ELISA. This group included 38 patients that manifested either relapses of established, clinically definite MS or first attacks of demyelinating disease, primarily in the form of optic neuritis with abnormal magnetic resonance imaging (MRI) brain scan and/or abnormal CSF. These patients are designated as MS cases in Table 1. Diagnoses were based on published criteria for clinical research (41-44). No patient received immunomodulatory therapy including corticosteroids before LP; mean time from symptom onset to LP was 14 days. Control subjects $(n=31)$ in the CSF chemokine ELISA study were divided into two groups (Table 1$)$ : controls (CON; $n=21)$, who had no CNS or major systemic disorder, and other neurological disease controls (OND; $n=10$ ), who had CNS disorders.

The second group of patients underwent LP and venipuncture for analysis of chemokine receptors on cells in CSF and blood. In this group, all patients $(n=9)$ manifested relapses of established MS. LP and venipuncture were also performed during diagnostic evaluation on patients with aseptic meningitis $(n=5)$. CSF in excess of that needed for diagnosis was analyzed for chemokine receptors on $\mathrm{T}$ cells. For determination of chemokine receptors on circulating cells, venipuncture was performed on healthy volunteers.

CSF processing and routine analysis. CSF was immediately chilled and centrifuged at $250 \mathrm{~g}$ for $10 \mathrm{~min}$ to remove cells. Supernatants were snap-frozen within $20 \mathrm{~min}$ of lumbar puncture and maintained at $-80^{\circ} \mathrm{C}$ until analysis.

CSF cell counts were determined with a hemocytometer. 
Albumin was measured by rate nephelometry (Array Protein System; Beckman Instruments Inc., Brea, California, USA). Upper limits of normal were four leukocytes per microliter of CSF. For patients under the age of 45 years, normal CSF/serum albumin ratio was 0.0068 , while above that age it was 0.0102 . $\mathrm{CSF} /$ serum albumin ratios were used to evaluate the integrity of the $\mathrm{BBB}$, which normally excludes this serum protein from the CNS. Oligoclonal immunoglobulin bands were detected by isoelectric focusing and immunoblotting (45).

ELISA. Chemokine concentrations were determined by ELISA on coded samples, as described previously (46). Importantly, these assays have been extensively validated on human body fluids from several sites, including the nervous system (46). Chemokine concentrations were measured twice in separate coded ELISA runs on 21 randomly selected CSF specimens, with an interassay variability below $10 \%$.

Brain tissues. Studies were performed on brain tissue taken at autopsy from eight patients with MS and two controls (see Table 3). In the MS material, 14 demyelinated lesions (12 paraffin-embedded tissue samples from six brains, and two freshfrozen samples from two other brains) were identified by staining for myelin proteins and by hematoxylin-eosin/Luxol fast blue histochemistry. The study was focused on lesions that were actively demyelinating according to established criteria specifying the presence of macrophages containing myelin debris. Furthermore, all lesions were actively inflamed, exhibiting perivascular leukocyte infiltrates $(1,47)$.

Antibodies. The following antibodies were used for immunohistochemistry: murine monoclonal anti-CXCR3 (LeukoSite Inc., Cambridge, Massachusetts, USA) (48), goat polyclonal antiCXCR3 (Santa Cruz Biotechnology Inc., Santa Cruz, California, USA), murine monoclonal anti-CCR5 (clone 183; R\&D Systems Inc., Minneapolis, Minnesota, USA), goat polyclonal anti-CCR5 (Santa Cruz Biotechnology Inc.), rabbit polyclonal anti-CCR5 (kindly provided by W. Marasco, Dana-Farber Cancer Institute, Boston, Massachusetts, USA) (49), murine monoclonal anti-CCR1 (LeukoSite Inc.), goat polyclonal anti-CCR1 (Santa Cruz Biotechnology Inc.), goat polyclonal anti-CCR3 (Santa Cruz Biotechnology Inc.), and murine monoclonal anti-IP-10 (LeukoSite Inc.) (50).

Immunohistochemistry. Formalin-fixed, paraffin-embedded tissue sections were placed on Superfrost Plus slides (Fisher Scientific Co., Pittsburgh, Pennsylvania, USA), deparaffinized in xylenes, and rehydrated through graded ethanol into PBS. Antigen retrieval was performed by microwaving in $1 \mathrm{mM}$ EDTA, $\mathrm{pH}$ 8.0 (51). Frozen tissues, sectioned with a cryostat onto Superfrost Plus slides, were dried, stored at $-70^{\circ} \mathrm{C}$, and fixed in icecold acetone immediately before staining.

All immunostaining procedures were performed with the avidin-biotin-horseradish peroxidase complex procedure and 3,3-diaminobenzidine (DAB; Sigma Chemical Co., St. Louis, Missouri, USA) as described previously (1). Slides were treated with $1 \% \mathrm{H}_{2} \mathrm{O}_{2}$ followed by $10 \%$ human serum, incubated overnight with primary antibodies at $4{ }^{\circ} \mathrm{C}$, washed, incubated with biotiny- lated secondary antibody at room temperature for $30 \mathrm{~min}$, washed, and incubated with avidin-biotin-horseradish peroxidase complex (Vectastain Elite; Vector Laboratories, Burlingame, California, USA), according to the manufacturer's instructions. After development with DAB substrate, slides were mounted in $80 \%$ glycerol in PBS. For each antibody, optimal concentrations of primary and secondary antibody were determined empirically.

Preparation of cells for flow cytometry. Cells were prepared for analysis on a FACS ${ }^{\circledR}$ (Becton Dickinson Immunocytometry Systems, San Jose, California, USA) as described previously (52). Aliquots $(12 \mathrm{ml})$ of CSF were collected directly on ice. After cells were counted in a Jessen Chamber, CSF was centrifuged immediately at $250 \mathrm{~g}$ for $10 \mathrm{~min}$ at $4^{\circ} \mathrm{C}$. The supernatant was removed, and cells were washed once in PBS containing $1 \%$ BSA and $0.1 \%$ sodium azide (PBS/BSA/azide) and resuspended in ice-cold PBS/BSA/azide at a concentration of 40,000 mononuclear cells per milliliter. Blood mononuclear cells were obtained from heparinized venous blood by density gradient centrifugation on Lymphoprep (Nycomed, Oslo, Norway), washed three times in HBSS with $0.2 \%$ human serum albumin, and resuspended in PBS/BSA/azide at a concentration of 1 million mononuclear cells per milliliter.

To detect CXCR3 on lymphocyte subsets, $100 \mu \mathrm{l}$ of CSF cells (at least 4,000 mononuclear cells) or blood cell suspensions $(100,000$ mononuclear cells) were incubated for $30 \mathrm{~min}$ with anti-CXCR3 (Clone 49801.111; R\&D Systems Inc.) or IgG1 isotype control antibody (Clone 11711.11; R\&D Systems Inc.). After one wash and resuspension in FACS/PBS, cells were incubated with FITC-conjugated anti-mouse IgG1 (Clone A85-1; PharMingen, San Diego, California, USA) for $30 \mathrm{~min}$. After a second wash in PBS/BSA/azide, the cells were incubated with peridinin chlorophyll protein (PerCP)-conjugated anti-CD8 (Clone SK1) or allophycocyanin (APC)-conjugated anti-CD4 antibody (Clone SK3) (Becton Dickinson Immunocytometry Systems) for $30 \mathrm{~min}$. Stained cells were then washed twice in PBS/BSA/azide, fixed with $1 \%$ paraformaldehyde, and stored at $4^{\circ} \mathrm{C}$ until analysis. For CCR5 detection on lymphocyte subsets, the following were used: FITC-conjugated anti-CCR5 (Clone 2D7/CCR5; Becton Dickinson Immunocytometry Systems), PerCP-conjugated anti-CD8, and APC-conjugated anti-CD4. The following isotype controls were used: FITC-, phycoerythrin (PE)-, or PerCP-conjugated mouse IgG1 (Clone MOPC-21; Becton Dickinson Immunocytometry Systems), and APC-conjugated anti-CD3 (Clone SK7; Becton Dickinson Immunocytometry Systems). The cells were stained for $30 \mathrm{~min}$ in darkness at $4^{\circ} \mathrm{C}$, washed twice in $\mathrm{PBS} / \mathrm{BSA} /$ azide, and fixed in $1 \%$ paraformaldehyde before analysis. Samples were analyzed on a four-color FACSCalibur flow cytometer (Becton Dickinson Immunocytometry Systems). For data analysis, $\mathrm{CD} 4$ or $\mathrm{CD} 8$ positive lymphocytes were gated, and the marker was set at $1 \%$ isotype-control positive events.

Statistics. Differences between group CSF chemokine levels were analyzed with one-way ANOVA, using Bonferroni's post hoc test for multiple comparisons; $P<0.05$ was considered signifi-

Table 2

Chemokine levels $(\mathrm{pg} / \mathrm{ml} \pm \mathrm{SEM})$ in CSF of MS patients and controls

\begin{tabular}{|c|c|c|c|c|c|c|c|}
\hline \multirow[b]{2}{*}{ Diagnosis } & \multicolumn{2}{|c|}{$\begin{array}{l}\text { Non-ELRA } \alpha \text {-chemokines: } \\
\text { T-cell chemoattractants }\end{array}$} & \multicolumn{2}{|c|}{$\begin{array}{l}\text { ELR } \alpha \text {-chemokines: } \\
\text { neutrophil chemoattractants }\end{array}$} & \multicolumn{3}{|c|}{$\begin{array}{c}\beta \text {-chemokines: } \\
\text { T-cell and monocyte chemoattractants }\end{array}$} \\
\hline & IP-10 & Mig & GRO-a & IL-8 & RANTES & MCP-1 & MIP-1 $\alpha$ \\
\hline $\mathrm{MS}(n=38)$ & $936 \pm 72$ & $620 \pm 32$ & $31 \pm 3$ & $50 \pm 4$ & $103 \pm 16$ & $536 \pm 36$ & ND \\
\hline $\operatorname{CON}(n=21)$ & $319 \pm 43$ & $464 \pm 36$ & $30 \pm 6$ & $29 \pm 10$ & $2 \pm 2$ & $745 \pm 33$ & ND \\
\hline OND $(n=10)$ & $586 \pm 229$ & $574 \pm 106$ & $48 \pm 11$ & $36 \pm 10$ & $40 \pm 26$ & $801 \pm 56$ & ND \\
\hline MS vs. CON & $P<0.001$ & $P<0.05$ & NS & NS & $P<0.001$ & $P<0.001$ & - \\
\hline MS vs. OND & NS & NS & NS & NS & NS & $P<0.001$ & - \\
\hline
\end{tabular}

${ }^{\mathrm{A} G l u t a m a t e-l e u c i n e-a r g i n i n e ~ m o t i f ~ i n ~ t h e ~} \mathrm{NH}_{2}$-terminal domain. ${ }^{\mathrm{B}} \mathrm{ng} / \mathrm{ml} \mathrm{CSF} \pm$ standard error of the mean. $\mathrm{ND}$, not detected at assay sensitivity of $10 \mathrm{pg} / \mathrm{ml}$; $\mathrm{NS}$, not 


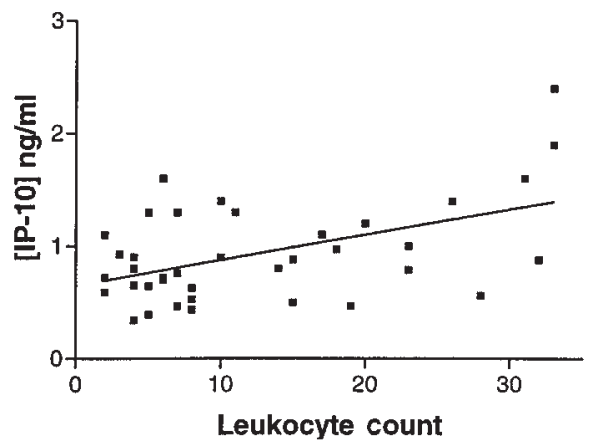

Figure 1

IP-10 concentration correlates with leukocyte count in CSF of MS patients during acute attacks. The scatter diagram shows the relationship between concentration of IP-10 and leukocyte count in the CSF of MS patients ( $n=$ 38). Each closed square represents data from one patient. The best-fit line is shown. Pearson $r$ value $=0.4925$; two-tailed $P=0.0017$. CSF, cerebrospinal fluid; IP-10, interferon- $\gamma$-inducible protein of $10 \mathrm{kDa} ; \mathrm{MS}$, multiple sclerosis.

cant. The Pearson $r$ value was calculated to analyze correlations between CSF cell counts and chemokine levels. For chemokine receptor expression on $\mathrm{T}$ cells, differences between blood and CSF were analyzed by paired $t$ test.

\section{Results}

CSF IP-10, Mig, and RANTES levels are elevated in patients with $M S$ attacks. Using enzyme-linked immunosorbent assay (ELISA), we determined CSF levels of seven chemokines IP-10, Mig, RANTES, MCP-1, MIP- $1 \alpha$, interleukin-8 (IL$8)$, and growth-regulated oncogene- $\alpha($ GRO- $\alpha)-$ in MS patients and controls (Table 1). Thirty-eight MS patients were studied during symptomatic episodes of inflammatory demyelination. Of 31 controls, 21 (CON) had noninflammatory benign conditions and were considered free of CNS disease. Ten OND controls had varied CNS disorders, including spondylotic myelopathy, metastasis to the nervous system, or motor neuron disease. MS patients exhibited significantly increased levels of three chemokines (IP-10, Mig, and RANTES), compared with CON (Table 2). One OND control with carcinomatous meningitis had elevated intrathecal levels of multiple

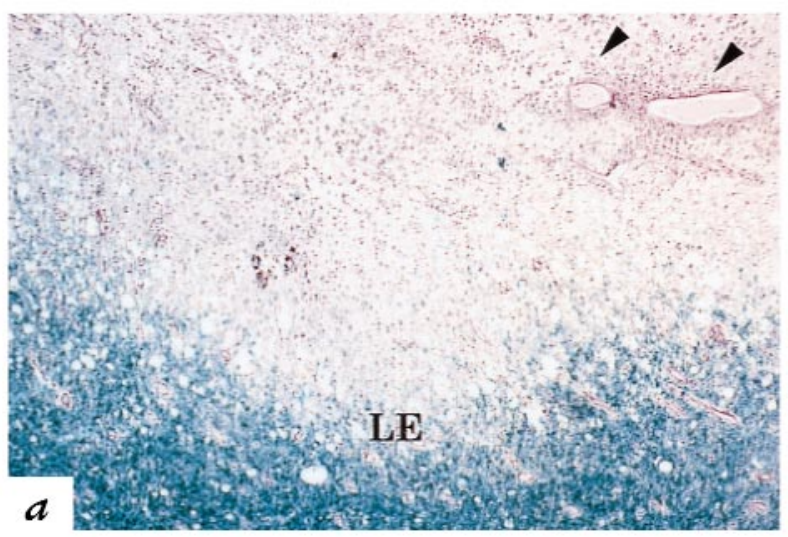

chemokines; one OND control with inflammatory polyradiculoneuropathy had very high CSF levels of Mig. Neither of these two OND controls exhibited patterns of CSF chemokine expression characteristic of MS patients.

IP-10 was detected in all MS and control CSF samples, with mean concentration increased nearly threefold in MS compared with CON patients (Table 2). Regression analysis indicated a significant linear relationship between CSF cell count and IP-10 concentration (Fig. 1). We considered whether the differences in IP-10 levels between MS and CON patients could be confounded by the significantly lower age of the MS patients (Table $1 ; P<0.01$ ). However, there was not a significant relationship between age and CSF IP-10 levels in CON patients (Spearman $\rho=0.025 ; P=$ 0.27). Furthermore, there was a tendency for IP-10 levels to rise with age, suggesting that the differences we observed would be increased by examining age-matched populations. Mean CSF Mig levels were elevated by approximately $50 \%$ in MS patients (Table 2). RANTES, undetectable in 20 of 21 CON patients, was identified in the CSF of $58 \%$ of patients with MS attacks, with a mean level in the physiologically relevant picomolar range (Table 2 ). Concentrations of Mig and RANTES did not significantly correlate with CSF cell counts. Levels of MCP-1 were significantly decreased in MS patients, compared with CON or OND. Levels of three other chemokines (MIP- $1 \alpha$, GRO- $\alpha$, IL-8) were not altered or below the range of detection (Table 2).

Expression of CXCR3 and IP-10 in MS brain lesions. CXCR3 is the IP-10/Mig receptor on activated T cells $(53,54)$. When sections from inflammatory demyelinating MS brain lesions (Fig. 2, $a$ and $b$ ) (47) were analyzed with antiCXCR3 antibodies, small, round CXCR3-immunoreactive cells were observed in perivascular leukocyte cuffs (Fig. 3a). CXCR3-positive cells, morphologically consistent with lymphocytes, were similar in appearance and distribution to cells detected with the pan-T-cell marker anti-CD3 (Fig. 3c). We readily identified CXCR3-positive cells in perivascular cuffs from 14 of 14 lesions present in eight separate MS autopsy brains (Table 3).

To address the frequency with which CXCR3-positive cells were present in MS perivascular infiltrates, all $(n=$ $205)$ perivascular cuffs in the four largest MS lesions from

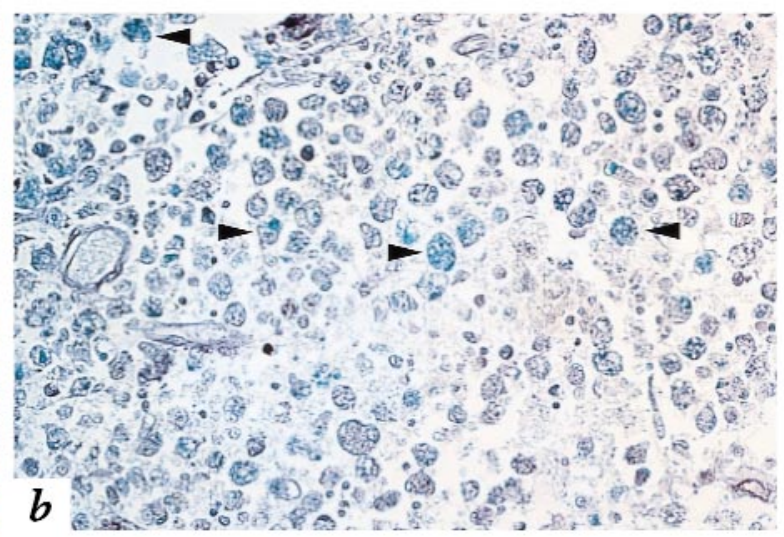

Figure 2

Inflammatory demyelination in an active MS lesion. (a) Hematoxylin-eosin histochemistry was combined with Luxol fast blue, which stains myelin blue. This technique reveals a zone of demyelination, abundant perivascular inflammation (arrowheads), and an irregular, hypercellular lesion edge $(L E)$, below which is normal white matter. $\times 80$. (b) Within the lesion, myelin debris, stained blue with Luxol fast blue, is evident within phagocytic macrophages (arrowheads), indicating active demyelination. $\times 170$. 

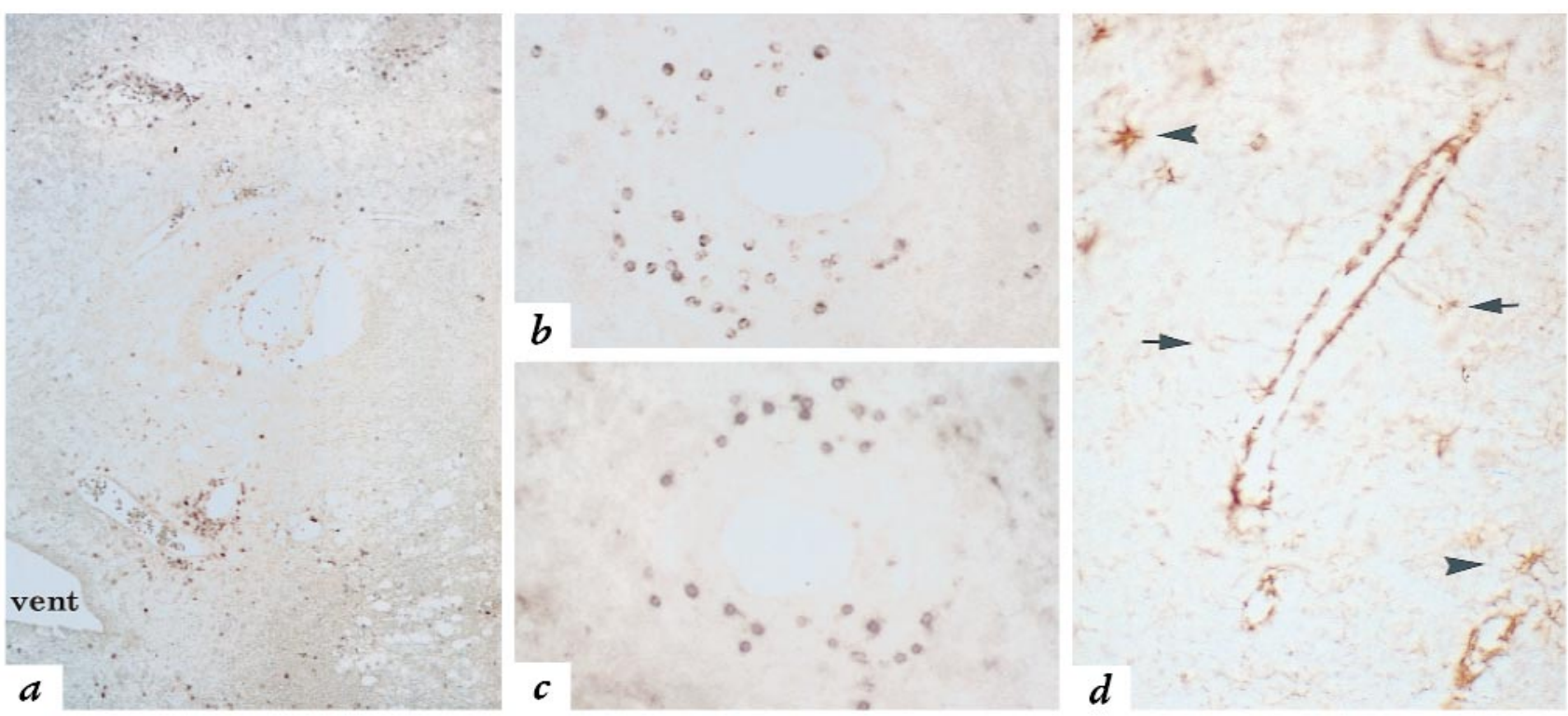

Figure 3

Expression of CXCR3 and IP-10 in MS lesions. (a) Numerous CXCR3-immunoreactive cells are present in perivascular inflammatory infiltrates in an acute MS lesion in white matter surrounding a ventricle $($ vent $) . \times 80 .(\boldsymbol{b})$ CXCR3-immunoreactive cells in a perivascular infiltrate exhibit similar morphology and distribution to CD3-positive T cells $(\boldsymbol{c}) . \times 170$. (d) Immunohistochemistry for IP-10 reveals staining of the cell bodies of reactive astrocytes (arrows). Intense staining is also seen in processes, consistent with astrocytic end-feet that extend to, and surround, a blood vessel. Scattered reactive astrocytes in the surrounding parenchyma also express IP-10 (arrowheads). $\times 300$. CXCR3, type 3 CXC chemokine receptor.

three different brains were identified under Nomarski optics and examined for CXCR3-positive cells. Abundant CXCR3-bearing cells were detected in 203 of 205 (99\%) perivascular infiltrates, typically comprising $5 \%-25 \%$ of perivascular mononuclear cells. CXCR3-positive cells were not detected in normal-appearing white matter (NAWM) of MS brain or in either of two control brains.

IP-10 immunoreactivity was identified in frozen sections from two autopsy specimens and one biopsy specimen, primarily associated with astrocytes around inflammatory lesions (Fig. 3d); the production of IP-10 by astrocytes was described previously in $\operatorname{EAE}(25,29,55)$. Intense staining was observed in astrocyte foot processes, structures that surround cerebral blood vessels and help maintain BBB integrity (Fig. 3d). IP-10 immunoreactivity was not evident in NAWM (not shown).

Distribution of RANTES receptors in MS brain lesions. RANTES, which was significantly elevated in MS CSF specimens (Table 2), engages diverse receptors, including CCR1, CCR3, and CCR5 (13). We examined expression of these receptors in MS lesions.

CCR5 expression is associated with T-helper 1 (Th1) functional differentiation of $\mathrm{T}$ cells $(53,56,57)$. Using both polyclonal and monoclonal antisera to make distinct portions of the molecule, CCR5 immunoreactivity was demonstrated on cells morphologically consistent with $T$ cells, phagocytic macrophages, and microglia in lesional areas of MS brain tissue (Fig. 4). CCR5-positive cells were identified in actively demyelinating MS lesions $(n=13)$ in all eight brains.

CCR1, expressed by a majority of circulating T cells and monocytes but not by B cells or neutrophils (58), responds to multiple chemokines, including RANTES. CCR1-positive cells were occasionally detected in the perivascular infiltrates of 5 of 12 acute MS lesions in 5 of 8 autopsied brains (Fig. 5a). CCR1-immunoreactive cells exhibited the morphology of small lymphocytes. Their infrequent detection was not due to technical considerations, because all tissues examined contained CCR1-positive cells within blood vessels. CCR3 expression is associated with Th2polarized T cells $(57,59)$. CCR 3 was detected in only one of the five largest acute MS lesions studied (Fig. $5 b$ ).

CXCR3-positive $\mathrm{CD}^{+} \mathrm{T}$ cells are enriched in CSF compared with peripheral blood. Observations described above implicated IP-10/Mig-CXCR3 signaling in MS-related inflammation. To address the functional significance of these findings in vivo, we quantitated CXCR3-bearing $\mathrm{T}$ cells in the peripheral blood and CSF of individual MS patients. CXCR3/CD4 double-positive cells in peripheral blood of six MS patients represented $38 \%$ of the $\mathrm{CD}^{+}$population, not significantly different from either neurological controls (not shown) or healthy controls, and consistent with published values (Table 4) (48). In MS CSF, $80 \%$ of CD4 ${ }^{+}$ cells expressed CXCR3, a significant enrichment compared with peripheral blood $(P=0.004$; Table 4$)$. These

Table 3

Chemokine receptor analysis in CNS tissues: patient characteristics

$\begin{array}{lcccccc}\text { ID number } & \begin{array}{c}\text { Age Gender } \\ \text { (years) }\end{array} & \begin{array}{c}\text { Autopsy } \\ \text { time (h) }\end{array} & \text { Diagnosis } & \begin{array}{c}\text { Number } \\ \text { of lesions }\end{array} & \text { Source } \\ 35 \text { Y94-3N/N1 } & 29 & \mathrm{~F} & 17 & \text { MS } & 1 & \text { Mayo } \\ \text { W90-37 } & 42 & \mathrm{M} & 18 & \text { MS } & 1 & \text { Wistar } \\ \text { YR95-88/5,8 } & 47 & \text { M } & \text { unk } & \text { MS } & 2 & \text { Mayo } \\ 2684 & 51 & \mathrm{~F} & 6 & \text { MS } & 1 & \text { NNRSB } \\ 2765 & 51 & \mathrm{~F} & 9 & \text { MS } & 1 & \text { NNRSB } \\ \text { W90-19 } & 60 & \mathrm{~F} & \text { unk } & \text { MS } & 5 & \text { Wistar } \\ 3 \text { 385-N321; W8 } & 69 & \mathrm{~F} & 2 & \text { MS } & 2 & \text { Mayo } \\ \text { W90-22 } & 80 & \mathrm{~F} & 4 & \text { MS } & 1 & \text { Wistar } \\ 2607 & 65 & \mathrm{M} & 26 & \text { Non-neurological } & - & \text { NNRSB } \\ 2601 & 86 & \mathrm{M} & 13 & \text { Non-neurological } & - & \text { NNRSB }\end{array}$

ABrain examined at Mayo (autopsy performed elsewhere). CNS, central nervous system; NNRSB, National Neurological Research Specimen Bank; unk, unknown. 

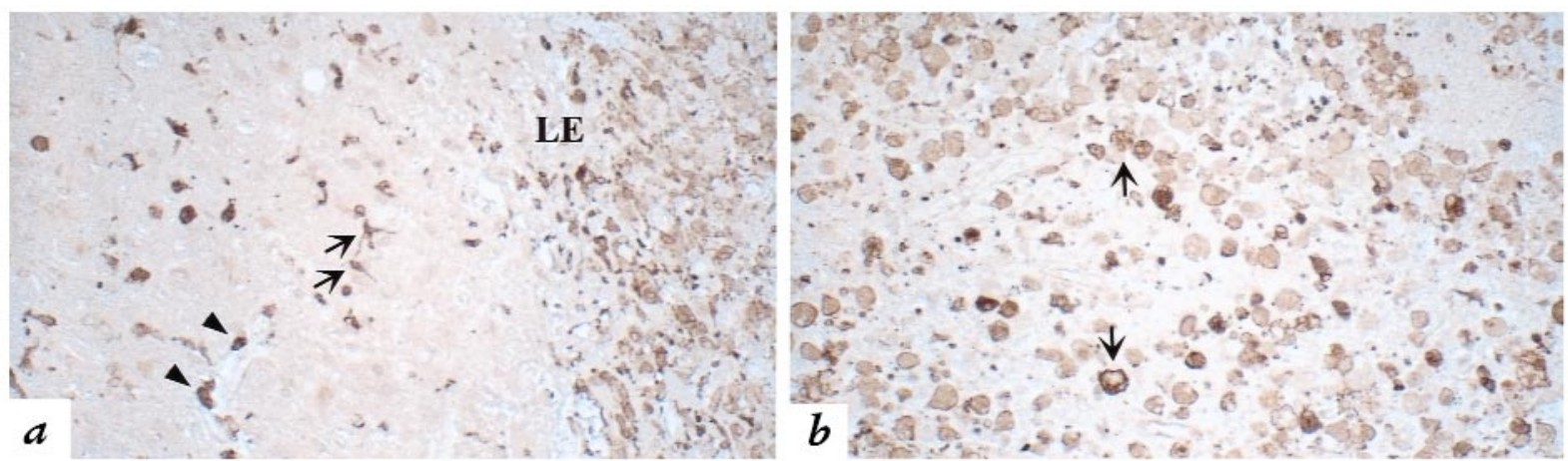

Figure 4

CCR5 expression by multiple leukocyte populations in an active MS lesion. (a) Near the lesion edge (LE), CCR5 immunohistochemistry reveals scattered elongated process-bearing cells consistent with reactive microglia (arrows) and small, round lymphocytic perivascular cells (arrowheads), while the lesion (the portion of the panel to the right of the lesion edge) contains numerous CCR5-positive phagocytic macrophages. $\times 170$. (b) CCR5-immunoreactive cells in the lesion center are primarily phagocytic macrophages (arrows). $\times 170$. CCR5, type 5 CC chemokine receptor.

results supported the functional significance of IP10/Mig-CXCR3 interactions in recruiting CD4+ $\mathrm{T}$ cells from the circulation to the intrathecal compartment of MS patients. Eighty percent of circulating CD8 ${ }^{+}$cells from MS patients were CXCR3-positive, not significantly different from controls. Ninety-seven percent of MS CSF $\mathrm{CD}^{+}$cells expressed CXCR3 $(P=0.044$; Table 4$)$.

Similar findings concerning CXCR3 expression for both $\mathrm{CD}^{+}$and $\mathrm{CD}^{+}$cells were obtained in analysis of paired blood and CSF samples from five patients with aseptic meningitis (data not shown). This finding was not surprising: meningitis CSF contains T-cell chemotactic activity that is neutralized by anti-IP-10 antibodies. Furthermore, it has been reported that the activatedmemory phenotype of CSF T cells in MS and aseptic meningitis is not distinguishable $(60,61)$.

CCR5-positive Tcells are enriched in CSF compared with peripheral blood. To address the functional significance of elevated CSF RANTES and CCR5 expression in MS brain lesions, we compared the percentages of $\mathrm{CD}^{+}$and $\mathrm{CD} 8^{+} \mathrm{T}$ cells that expressed CCR5 in the blood and CSF of seven MS patients. Circulating CD8 ${ }^{+}$cells in MS patients were $15 \%$ CCR5-positive, significantly increased compared with healthy indi-

Table 4

CXCR3 expression ${ }^{\mathrm{A}}$ by CD4 and CD 8 cells in blood and CSF

\begin{tabular}{|c|c|c|c|c|c|c|c|}
\hline \multirow[b]{2}{*}{ Patient ID } & \multicolumn{4}{|c|}{ CD4 } & \multicolumn{3}{|c|}{ CD8 } \\
\hline & Diagnosis & Blood & Significance & $\mathrm{CSF}$ & Blood & Significance & CSF \\
\hline 758 & MS & 22 & & 87 & 81 & & 98 \\
\hline 759 & MS & 27 & & 74 & 76 & & 95 \\
\hline 760 & MS & 44 & & 79 & 99 & & 99 \\
\hline 772 & MS & 24 & & 72 & 64 & & 98 \\
\hline 773 & MS & 69 & & 84 & 97 & & 100 \\
\hline 774 & MS & 44 & & 85 & 98 & & 99 \\
\hline 776 & MS & 36 & & 73 & 44 & & 93 \\
\hline Mean \pm SEM & & $38 \pm 6$ & $P=0.004^{B}$ & $79 \pm 2$ & $80 \pm 8$ & $P=0.044^{B}$ & $97 \pm 1$ \\
\hline $\mathrm{H} 1$ & healthy & 58 & & nd & 92 & & nd \\
\hline $\mathrm{H} 2$ & healthy & 58 & & nd & 84 & & nd \\
\hline $\mathrm{H} 3$ & healthy & 49 & & nd & 80 & & nd \\
\hline $\mathrm{H} 4$ & healthy & 72 & & nd & 92 & & nd \\
\hline H5 & healthy & 20 & & nd & 77 & & nd \\
\hline $\mathrm{H} 6$ & healthy & 27 & & nd & 79 & & nd \\
\hline Mean \pm SEM & & $47 \pm 8$ & $\mathrm{NS}^{\mathrm{C}}$ & - & $84 \pm 3$ & $\mathrm{NS}^{\mathrm{C}}$ & - \\
\hline
\end{tabular}

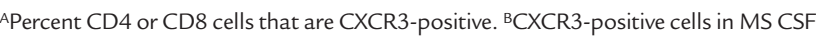
compared with MS blood. 'CXCR3-positive circulating $\mathrm{CD}^{+}{ }^{+}$or $\mathrm{CD} 8{ }^{+}$cells in control compared with MS subjects. NS, not significant; $n d$, not done. viduals $(P=0.005$; Table 5$)$. CD8 ${ }^{+}$cells in MS CSF were further enriched (38\%) for CCR5 expression compared with peripheral blood $\left(P=0.017\right.$; Table 5). Circulating $\mathrm{CD}^{+}{ }^{+}$cells in MS patients expressed CCR5 at a low level (4\%), with a significant increase to $13 \%$ in $\operatorname{CSF}(P=0.018$; Table 5$)$.

\section{Discussion}

To address mechanisms of inflammatory CNS pathogenesis and identify putative molecular targets for therapeutic intervention, we evaluated chemokines and their receptors in MS. In this report, we focus on studies performed on patients with active disease, in the form of either acute symptomatic attacks or inflammatory demyelinating lesions in autopsied brain (47). We found consistent alterations of two ligand-receptor systems: IP10/Mig-CXCR3 and RANTES-CCR5. These abnormalities included elevated chemokine ligand concentrations in CSF, with corresponding enrichment of receptor-bearing cells in the intrathecal compartment. The distribution and abundance of receptor-bearing cells in affected CNS tissues implied pathogenic significance for the findings of increased CSF chemokine.

CSF chemokine levels are altered during attacks of symptomatic inflammatory demyelination. To initiate studies of chemokine expression in MS, we analyzed CSF, which reflects the composition of the extracellular interstitial fluid of CNS white matter (62). The BBB was largely intact in more than $95 \%$ of the MS patients in this study (Table 1); therefore, the elevated CSF chemokine levels most likely reflected intrathecal chemokine production rather than diffusion from blood.

CSF levels of RANTES, Mig, and IP-10 were significantly elevated in MS (Table 2), and there was a positive correlation between the concentration of IP-10 and CSF cell count (Fig. 1). Other chemokines that act toward monocytes and T cells, including MIP-1 $\alpha$, MIP- $1 \beta$ (not shown), and MCP-1, were either undetectable or modestly reduced during MS attacks (Table 2). MIP- $1 \alpha$ was previously reported to be slightly elevated in MS subjects, with a mean level of $4 \mathrm{pg} / \mathrm{ml}$ (40). Because the lower limit of detection in our ELISA was $10 \mathrm{pg} / \mathrm{ml}$, failure to find measurable CSF MIP- $1 \alpha$ was consistent with this prior report. We did not detect the related chemokine MIP-1 $\beta$ in a representative subset of 20 CSF samples (15 
MS, 5 CON; data not shown). The neutrophil-directed $\alpha$-chemokines IL- 8 and GRO- $\alpha$ were not altered during the acute MS disease process.

Published data concerning CSF chemokine levels have been obtained primarily from studying infectious disorders. Patients with aseptic/viral meningitis and mononuclear pleocytosis had increased levels of chemokines, such as MCP-1, MIP-1 $\alpha$, and IP-10 $(11,61,63)$. In aseptic meningitis, CSF cell counts correlated well with chemokine levels, and chemotactic activity of CSF was abolished by anti-chemokine antibodies $(61,63,64)$. Elevated chemokine levels in the CSF of MS patients did not reflect nonspecific inflammatory change in the intrathecal compartment: RANTES was elevated in MS CSF but undetectable in either viral or bacterial meningitis subjects $(61,63,65)$. MCP-1 levels were markedly elevated in CSF from meningitis and HIV dementia patients but moderately depressed (Table 2) during episodes of inflammatory demyelination $(61,63,65,66)$.

It was unexpected to observe reduced CSF MCP-1 levels in MS patients undergoing attacks: MCP-1 is expressed by astrocytes bordering MS lesions $(38,39)$, and robust CNS MCP-1 expression has been repeatedly observed during attacks of EAE. Moreover, anti-MCP-1 antibodies suppressed EAE relapses (22-25). Reduced CSF MCP-1 levels during acute attacks could provide new insight into immunoregulatory characteristics of MS. In particular, it has been proposed that MS relapses are triggered by predominance of Th1, as compared with Th2, lymphokines (67, 68). MCP-1 exposure influences $T$ cells toward Th2 commitment, and MCP-1 expression is regulated by IL4 , the cardinal Th2 cytokine (69-71). We speculate that reduced CSF MCP-1 concentrations corresponded to increased Th1 activity during MS attacks, as reported recently (67). It is also notable that RANTES, IP-10, and Mig, associated with in vitro activity toward activated Th1-like T cells, were most significantly elevated during acute MS attacks $(57,72)$. Our study patients were examined during acute attacks, and it is possible that chemokine levels at other stages of disease may prove to be substantially different.

Our findings in MS patients extend previous observa-

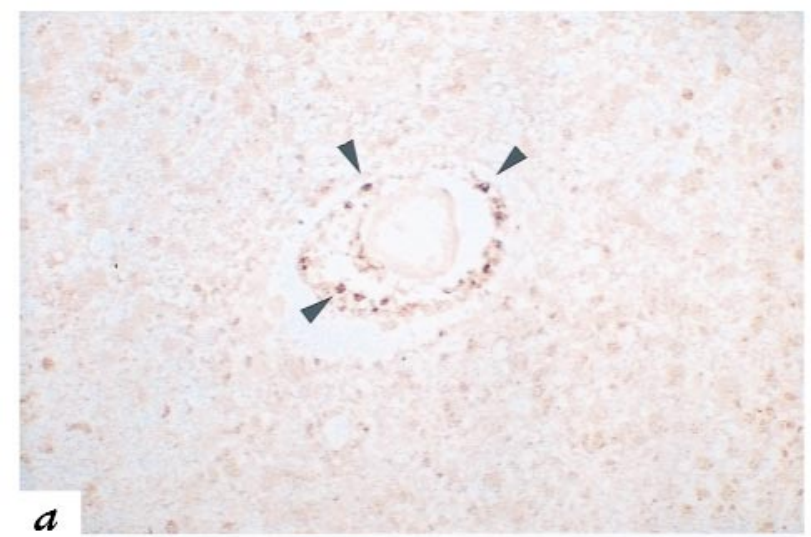

Table 5

CCR5 expression ${ }^{A}$ by CD4 and CD8 cells in blood and CSF

\begin{tabular}{|c|c|c|c|c|c|c|c|}
\hline \multirow[b]{2}{*}{ Patient ID } & \multirow[b]{2}{*}{ Diagnosis } & \multicolumn{3}{|c|}{ CD4 } & \multicolumn{3}{|c|}{ CD8 } \\
\hline & & Blood & Significanc & e CSF & Blood & Significance & CSF \\
\hline 758 & MS & 3 & & 11 & 14 & & 28 \\
\hline 759 & MS & 3 & & 6 & 17 & & 34 \\
\hline 760 & MS & 4 & & 8 & 19 & & 44 \\
\hline 762 & MS & 3 & & 6 & 18 & & 16 \\
\hline 767 & MS & 6 & & 15 & 13 & & 31 \\
\hline 773 & MS & 7 & & 31 & 14 & & 72 \\
\hline 776 & MS & 1 & & 15 & 9 & & 40 \\
\hline \multicolumn{2}{|l|}{ Mean \pm SEM } & $4 \pm 1$ & $P=0.018^{B}$ & $13 \pm 3$ & $15 \pm 1$ & $P=0.017^{B}$ & $38 \pm 7$ \\
\hline $\mathrm{H} 1$ & healthy & 2 & & nd & 7 & & nd \\
\hline $\mathrm{H} 2$ & healthy & 2 & & nd & 3 & & nd \\
\hline $\mathrm{H} 3$ & healthy & 2 & & nd & 11 & & nd \\
\hline $\mathrm{H} 4$ & healthy & 1 & & nd & 2 & & nd \\
\hline $\mathrm{H} 5$ & healthy & 4 & & nd & 8 & & nd \\
\hline $\mathrm{H} 6$ & healthy & 3 & & nd & 9 & & nd \\
\hline \multicolumn{2}{|c|}{ Mean \pm SEM } & $2 \pm 0.4$ & NSC & - & $7 \pm 1.4$ & $P=0.005^{C}$ & - \\
\hline
\end{tabular}

APercent CD4 or CD8 cells that are CCR5-positive. ${ }^{\mathrm{B} C C R} 5$-positive cells in MS CSF compared with MS blood. ${ }^{C} \mathrm{CCR} 5$-positive circulating $\mathrm{CD} 4{ }^{+}$or $\mathrm{CD} 8{ }^{+} \mathrm{T}$-cells in control compared with MS subjects.

tions of CSF chemokines in biologically meaningful (nanomolar) concentrations in controls and patients with CNS disorders (Table 2; and refs. 61, 63-66). Because the composition of the CSF reflects the extracellular interstitial fluid of the white matter, it is most plausible that CNS chemokines originate from cells in the perivascular subarachnoid space and the adjacent parenchyma. Consistent with this concept, we found that IP-10 immunoreactivity was particularly concentrated in astrocyte perivascular foot processes (Fig. $3 d$ ). Production of chemokines by leukocytes and resident CNS cells near inflamed vessels was described previously in EAE and has recently been reported in MS (25, 28, 37-39).

CNS chemokine receptor expression supports the functional significance of elevated CSF chemokine levels. We found that CXCR3 was the predominant chemokine receptor on lymphocytic cells in both CSF and parenchymal compartments, while CCR5 was present on a minority of lymphocytes in both brain and CSF. These findings support the possibility that activated $\mathrm{T}$ cells expressing CXCR3 preferentially gain access to CNS tissues during formation of acute MS lesions. In contrast, in rheuma-

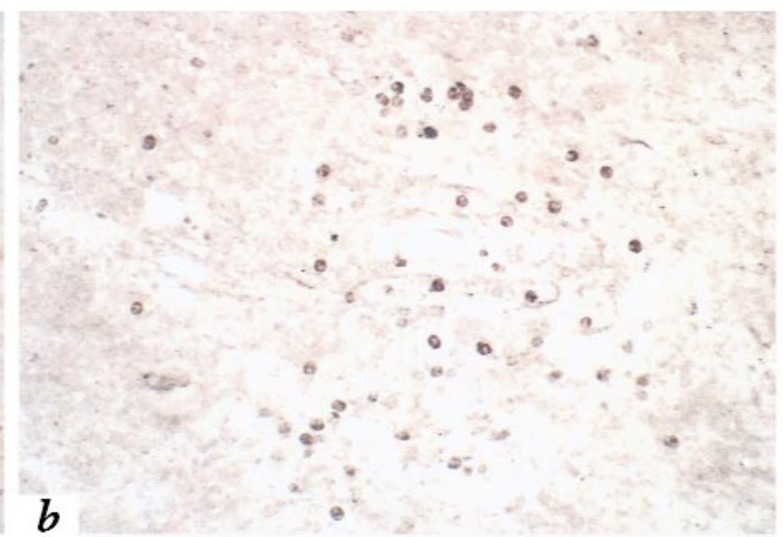

Figure 5

Expression of CCR1 and CCR3 in MS lesions. (a) CCR1 immunohistochemistry in an active lesion reveals small, round cells in the perivascular space. Immunoreactive cells are morphologically consistent with infiltrating lymphocytes. $\times 170$. (b) Small, round CCR3-immunoreactive cells in a highly active lesion are scattered through perivascular and parenchymal sites. $\times 170$. 
toid arthritis, the vast majority (more than $80 \%$ ) of infiltrating cells in synovial fluid expressed both CCR5 and CXCR3 (48). Although coexpression of chemokine receptors was not addressed in the current studies, such double-positive cells could constitute no more than a minority of the infiltrating population. The relatively modest enrichment of CCR5-positive cells in MS CSF is therefore consistent with the recent observation that individuals homozygous for nonfunctional mutant forms of CCR5 remain susceptible to MS (73).

Given elevated levels of IP-10 and Mig in MS CSF, it was of interest to determine whether cells bearing cognate receptors accumulated in the CSF. By monitoring expression of CXCR3 on T-cell subsets in blood and CSF, we found that $80 \%$ of CD $4+$ cells in MS CSF expressed CXCR3, suggesting that these receptor-bearing cells play a salient role in MS pathogenesis. The functional relevance of elevated CSF IP10 and Mig levels in MS (Table 2) was further supported by the uniform detection of CXCR3-positive cells in perivascular inflammatory cuffs in MS brain sections. These data are consistent with previous reports showing that anti-IP10 antibodies depleted the CSF of meningitis patients of chemotactic activity toward T-cell blasts (61). Interestingly, subarachnoid infusion of antisense IP-10 oligodeoxynucleotides reduced EAE disease activity (74).

CCR5, present on a minority of $\mathrm{T}$ cells, was highly expressed on phagocytic macrophages and microglia in actively demyelinating lesions (Fig. 4). Given these findings, engagement of CCR5 may be implicated in activating terminal effectors in the lesions of MS (75). CCR5 was expressed on a relatively restricted population of circulating $\mathrm{CD}^{+}$and $\mathrm{CD}^{+}$cells, with significant enrichment in the CSF. This observation agrees with CSF levels of RANTES, which were significantly elevated over normal concentration, but considerably lower in absolute concentration, than those observed for IP-10 or Mig (Table 2). Although alternative RANTES receptors, such as CCR1 and CCR3, were detected less frequently in the active MS lesions reported here, the significance of these receptors on lesions of different stage or pathogenesis remains quite plausible (76).

CCR5 epitopes have been reported on neurons and astrocytes, as well as on microglia and leukocytes (77). The physiological significance of CCR5 on neurons remains uncertain: neither humans nor mice that lack functional CCR5 appear to be neurologically impaired (78). However, rat hippocampal neurons expressed CCR5 mRNA and demonstrated physiological responses to RANTES in vitro (79). In this report, CCR5 was not detected on neurons, using mouse monoclonal antibodies to the $\mathrm{NH}_{2}$-terminus of CCR5 and goat polyclonal antisera to a $\mathrm{COOH}$-terminal peptide to define CCR5-bearing cells.

In summary, we found consistent expression of specific chemokines and chemokine receptors during active MS. The finding of increased accumulation of CXCR3positive and CCR5-positive CSF cells imparted functional significance to elevated CSF levels of IP-10, Mig, and RANTES. The pathogenic importance of these data was supported by the uniform detection of CXCR3 and CCR5 on lymphocytic cells, macrophages, and microglia in MS brain lesions. Taken together, these data support the participation of specific chemokines and receptors in the pathogenesis of inflammation and tissue damage in MS and imply that these molecules should be considered as targets for therapeutic intervention.

\section{Acknowledgments}

We are grateful to Richard A. Rudick and Jar-Chi Lee (Cleveland Clinic Foundation) for critical evaluation of the manuscript and helpful suggestions with statistical analyses. We also thank Dana Gabuzda (Dana-Farber Cancer Institute) for advice about immunohistochemistry. This research was supported by the Williams Family Fund for Multiple Sclerosis Research, the National Institutes of Health (RO1-32151 to R.M. Ransohoff; KO8-01950 to M. Tani), and the Weiman Foundation.

1. Trapp, B., et al. 1998. Axonal transection in the lesions of multiple sclerosis. N. Engl.J. Med. 338:278-290.

2. Rudick, R., Cohen, J., Weinstock-Guttman, B., Kinkel, R., and Ransohoff, R. 1996. Management of multiple sclerosis. N. Engl. J. Med. 337:1604-1612.

3. Sørensen, T., and Ransohoff, R. 1998. Etiology and pathogenesis of multiple sclerosis. Semin. Neurol. 18:287-295.

4. Steinman, L. 1996. Multiple sclerosis: a coordinated immunological attack against myelin in the central nervous system. Cell. 85:299-302.

5. Rudick, R., et al. 1999. Cerebrospinal fluid abnormalities in a phase III trial of avonex (IFNB-1a) for relapsing multiple sclerosis. J. Neuroimmunol. 93:8-15.

6. Cannella, B., Cross, A.H., and Raine, C.S. 1993. Anti-adhesion molecule therapy in experimental autoimmune encephalomyelitis. J. Neuroimmunol. 46:43-56.

7. Antel, J., and Owens, T. 1993. The attraction of adhesion molecules. Ann. Neurol. 34:123-124.

8. Yednock, T.A., et al. 1992. Prevention of experimental autoimmune encephalomyelitis by antibodies against alpha4beta1 integrin. Nature. 356:63-66.

9. Engelhardt, B., Conley, F.K., Kilshaw, P.J., and Butcher, E.C. 1995. Lymphocytes infiltrating the CNS during inflammation display a distinctive phenotype and bind to VCAM-1 but not to MAdCAM-1. Int. Immunol. 7:481-491.

10. Butcher, E.C., and Picker, L.J. 1996. Lymphocyte homing and homeostasis. Science. 272:60-66.

11. Luster, A. 1998. Chemokines-chemotactic cytokines that mediate inflammation. N. Engl. J. Med. 338:436-445.

12. Rollins, B.J. 1997. Chemokines. Blood. 90:909-928.

13. Baggiolini, M. 1998. Chemokines and leukocyte traffic. Nature. 392:565-568.

14. Campbell, J.J., et al. 1998. Chemokines and the arrest of lymphocytes rolling under flow conditions. Science. 279:381-384.

15. Lira, S., Fuentes, M., Strieter, R., and Durham, S. 1997. Transgenic methods to study chemokine function in lung and central nervous system. Methods Enzymol. 287:304-318.

16. Tani, M., et al. 1996. Neutrophil infiltration, glial reaction and neurological disease in transgenic mice expressing the chemokine $\mathrm{N} 51 / \mathrm{KC}$ in oligodendrocytes. J. Clin. Invest. 98:529-539.

17. Bell, M.D., et al. 1996. Recombinant human adenovirus with rat MIP-2 gene insertion causes prolonged PMN recruitment to the murine brain. Eur. J. Neurosci. 8:1803-1811.

18. Fuentes, M., et al. 1995. Controlled recruitment of monocytes/macrophages to specific organs via transgenic expression of MCP-1. J. Immunol. 155:5769-5776.

19. Ransohoff, R., Glabinski, A., and Tani, M. 1996. Chemokines in immunemediated inflammation of the central nervous system. Cytokine Growth Factor Rev. 7:35-46.

20. Karpus, W., and Ransohoff, R. 1998. Chemokine regulation of experimental autoimmune encephalomyelitis: temporal and spatial expression patterns govern disease pathogenesis [comment]. J. Immunol. 161:2667-2671.

21. Karpus, W.J., et al. 1995. An important role for the chemokine macrophage inflammatory protein- $1 \alpha$ in the pathogenesis of the $\mathrm{T}$ cell-mediated autoimmune disease, experimental autoimmune encephalomyelitis. $J$. Immunol. 155:5003-5010.

22. Karpus, W.J., and Kennedy, K.J. 1997. MIP-1alpha and MCP-1 differentially regulate acute and relapsing autoimmune encephalomyelitis as well as Th1/Th2 lymphocyte differentiation. J. Leukoc. Biol. 62:681-687.

23. Berman, J., Guida, M., Warren, J., Amat, J., and Brosnan, C. 1996. Localization of monocyte chemoattractant peptide- 1 expression in the central nervous system in experimental autoimmune encephalomyelitis and trauma in the rat. J. Immunol. 156:3017-3023.

24. Godiska, R., Chantry, D., Dietsch, G.N., and Gray, P.W. 1995. Chemokine expression in murine experimental allergic encephalomyelitis. J. Neuroimmunol. 58:167-176.

25. Glabinski, A., Tani, M., Strieter, R., Tuohy, V., and Ransohoff, R. 1997. Synchronous synthesis of $\alpha$ - and $\beta$-chemokines by cells of diverse lineage in the central nervous system of mice with relapses of experimental autoimmune 
encephalomyelitis. Am.J. Pathol. 150:617-630.

26. Glabinski, A., Tani, M., Tuohy, V.K., Tuthill, R., and Ransohoff, R.M. 1996. Central nervous system chemokine gene expression follows leukocyte entry in acute murine experimental autoimmune encephalomyelitis. Brain Behav. Immun. 9:315-330.

27. Hulkower, K., et al. 1993. Expression of CSF-1, c-fms, and MCP-1 in the central nervous system of rats with experimental allergic encephalomyelitis. $J$. Immunol. 150:2525-2533.

28. Miyagishi, R., Kikuchi, S., Takayama, C., Inoue, Y., and Tashiro, K. 1997. Identification of cell types producing RANTES, MIP- $1 \alpha$ and MIP- $1 \beta$ in rat experimental autoimmune encephalomyelitis by in situ hybridization. $J$. Neuroimmunol.77:17-26.

29. Tani, M., et al. 1996. In situ hybridization analysis of glial fibrillary acidic protein mRNA reveals evidence of biphasic astrocyte activation during acute experimental autoimmune encephalomyelitis. Am. J. Pathol. 148:889-896.

30. Tani, M., and Ransohoff, R.M. 1994. Do chemokines mediate inflammatory cell invasion of the central nervous system parenchyma? Brain Pathol. 4:135-144.

31. Glabinski, A.R., Tani, M., Balasingam, V., Yong, V.W., and Ransohoff, R.M. 1996. Chemokine monocyte chemoattractant protein-1 (MCP-1) is expressed by astrocytes after mechanical injury to the brain. J. Immunol. 156:4363-436.

32. Grzybicki, D., et al. 1998. Expression of monocyte chemoattractant protein (MCP-1) and nitric oxide synthase-2 following cerebral trauma. Acta Neu ropathol. 95:98-103.

33. Liu, T., et al. 1993. Cytokine-induced neutrophil chemoattractant mRNA expressed in cerebral ischemia. Neurosci Lett. 164:125-128.

34. McTigue, D., et al. 1998. Selective chemokine mRNA accumulation in the rat spinal cord after contusion injury. J. Neurosci. Res. 53:368-376.

35. Ransohoff, R., and Tani, M. 1998. Do chemokines mediate leukocyte recruitment in post-traumatic CNS inflammation? Trends Neurosci. 21:154-159.

36. Gourmala, N.G., Buttini, M., Limonta, S., Sauter, A., and Boddeke, H.W. 1997. Differential and time-dependent expression of monocyte chemoattractant protein- $1 \mathrm{mRNA}$ by astrocytes and macrophages in rat brain: effects of ischemia and peripheral lipopolysaccharide administration. J. Neuroim munol. 74:35-44.

37. Hvas, J., et al. 1997. Perivascular T cells express the proinflammatory chemokine RANTES mRNA in multiple sclerosis lesions. Scand. J. Immunol. 46:195-203.

38. McManus, C., et al. 1998. MCP-1, MCP-2 and MCP-3 expression in multiple sclerosis lesions: an immunohistochemical and in situ hybridization study. J. Neuroimmunol. 86:20-29.

39. Simpson, J., Newcombe, J., Cuzner, M., and Woodroofe, M. 1998. Expression of monocyte chemoattractant protein- 1 and other $\beta$-chemokines by resident and inflammatory cells in multiple sclerosis lesions. J. Neuroimmunol. 84:238-249.

40. Miyagishi, R., Kikuchi, S., Fukazawa, T., and Tashiro, K. 1995. Macrophage inflammatory protein- $1 \alpha$ in the cerebrospinal fluid of patients with multiple sclerosis and other inflammatory neurological diseases. J. Neurol. Sci. 129:223-227.

41. Poser, C.M., et al. 1983. New diagnostic criteria for multiple sclerosis: guidelines for research protocols. Ann. Neurol. 13:227-231.

42. Francis, D.A. 1991. Demyelinating optic neuritis: clinical features and differential diagnosis. Br. J. Hosp. Med. 45:376-379.

43. McDonald, W.I. 1976. Diagnosis and management of optic neuritis. Trans. Ophthalmol. Soc. NZ. 28:11-17.

44. Frederiksen, J.L., Larsson, H.B., and Jensen, C. 1997. Serial gadoliniumenhanced MRI in untreated patients with acute optic neuritis: implications for natural history. Acta Neurol. Scand. 96:22-27.

45. Sellebjerg, F., and Christiansen, M. 1996. Qualitative assessment of intrathecal IgG synthesis by isoelectric focusing and immunodetection: interlaboratory reproducibility and interobserver agreement. Scand. J. Clin. Lab. Invest. 56:135-143

46. Verma, M.J., et al. 1997. Chemokines in acute anterior uveitis. Curr. Eye Res. 16:1202-1208.

47. Lassmann, H., Raine, C., Antel, J., and Prineas, J. 1998. Immunopathology of multiple sclerosis. J. Neuroimmunol. 86:213-218.

48. Qin, S., et al. 1998. The chemokine receptors CXCR3 and CCR5 mark subsets of T cells associated with certain inflammatory reactions. J. Clin. Invest. 101:746-754.

49. Vallat, A.V., et al. 1998. Localization of HIV-1 co-receptors CCR5 and CXCR4 in the brain of children with AIDS. Am. J. Pathol. 152:167-178.

50. Sasseville, V.G., et al. 1996. Chemokine expression in simian immunodeficiency virus-induced AIDS encephalitis. Am. J. Pathol. 149:1459-1467.

51. Morgan, J., Navabi, H., Schmid, K., and Jasani, B. 1994. Possible role of tissue-bound calcium ions in citrate-mediated high-temperature antigen retrieval. J. Pathol. 174:301-307.

52. Sellebjerg, F., Jensen, J., and Ryder, L.P. 1998. Costimulatory CD80 (B7-1) and CD86 (B7-2) on cerebrospinal fluid cells in multiple sclerosis. J. Newroimmunol. 84:179-187.
53. Loetscher, M., et al. 1996. Chemokine receptor specific for IP10 and mig: structure, function, and expression in activated T-lymphocytes. J. Exp. Med. 184:963-969.

54. Farber, J.M. 1997. Mig and IP-10: CXC chemokines that target lymphocytes. J. Leukoc. Biol. 61:246-257.

55. Ransohoff, R.M., et al. 1993. Astrocyte expression of mRNA encoding cytokines IP-10 and JE/MCP-1 in experimental autoimmune encephalomyelitis. FASEB J. 7:592-602.

56. Loetscher, P., et al. 1998. CCR5 is characteristic of Th1 lymphocytes. Nature. 391:344-345.

57. Sallusto, F., Lenig, D., Mackay, C.R., and Lanzavecchia, A. 1998. Flexible programs of chemokine receptor expression on human polarized T helper 1 and 2 lymphocytes. J. Exp. Med. 187:875-883.

58. Su, S.B., Mukaida, N., Wang, J., Nomura, H., and Matsushima, K. 1996. Preparation of specific polyclonal antibodies to a C-C chemokine receptor, CCR1, and determination of CCR1 expression on various types of leukocytes. J. Lenkoc. Biol. 60:658-666.

59. Sallusto, F., Mackay, C.R., and Lanzavecchia, A. 1997. Selective expression of the eotaxin receptor CCR3 by human $\mathrm{T}$ helper 2 cells. Science. 277:2005-2007.

60. Vrethem, M., et al. 1998. CD4 and CD8 lymphocyte subsets in cerebrospinal fluid and peripheral blood from patients with multiple sclerosis, meningitis and normal controls. Acta Neurol. Scand. 97:215-220.

61. Lahrtz, F., et al. 1997. Chemotactic activity on mononuclear cells in the cerebrospinal fluid of patients with viral meningitis is mediated by interferongamma inducible protein-10 and monocyte chemotactic protein-1. Eur.J. Immunol. 27:2484-2489.

62. Weller, R. 1998. Pathology of cerebrospinal fluid and interstitial fluid of the CNS: significance for Alzheimer disease, prion disorders and multiple sclerosis. J. Neuropathol. Exp. Neurol. 57:885-894.

63. Sprenger, H., et al. 1996. Chemokines in the cerebrospinal fluid of patients with meningitis. Clin. Immunol. Immunopathol. 80:155-161.

64. Ostergaard, C., et al. 1996. Interleukin-8 in cerebrospinal fluid from patients with septic and aseptic meningitis. Eur. J. Clin. Microbiol. Infect. Dis. 15:166-169.

65. Spanaus, K.S., et al. 1997. C-X-C and C-C chemokines are expressed in the cerebrospinal fluid in bacterial meningitis and mediate chemotactic activity on peripheral blood-derived polymorphonuclear and mononuclear cells in vitro. J. Immunol. 158:1956-1964.

66. Conant, K., et al. 1998. Induction of monocyte chemoattractant protein-1 in HIV-1 Tat stimulated astrocytes and elevation in AIDS dementia. Proc. Natl. Acad. Sci. USA. 95:3117-3121.

67. Calabresi, P.A., et al. 1998. ELI-spot of Th-1 cytokine secreting PBMC's in multiple sclerosis: correlation with MRI lesions. J. Neuroimmunol. 85:212-219.

68. Olsson, T. 1995. Critical influences of the cytokine orchestration on the outcome of myelin antigen-specific T-cell autoimmunity in experimental autoimmune encephalomyelitis and multiple sclerosis. Immunol. Rev. 144:245-268.

69. Rollins, B., Yoshimura, T., Leonard, E., and Pober, J. 1990. Cytokine-activated human endothelial cells synthesize and secrete a monocyte chemoattractant, MCP-1/JE. Am. J. Pathol. 136:1229-1233.

70. Lu, B., et al. 1998. Abnormalities in monocyte recruitment and cytokine expression in monocyte chemoattractant protein 1-deficient mice. J. Exp. Med. 187:601-608

71. Karpus, W., et al. 1997. Differential CC chemokine-induced enhancement of T helper cell cytokine production. J. Immunol. 158:4129-4136.

72. Bonecchi, R., et al. 1998. Differential expression of chemokine receptors and chemotactic responsiveness of type $1 \mathrm{~T}$ helper cells (Th1s) and Th2s. J. Exp. Med. 187:129-134.

73. Bennetts, B.H., Teutsch, S.M., Buhler, M.M., Heard, R.N., and Stewart, G.J. 1997. The CCR5 deletion mutation fails to protect against multiple sclerosis. Hum. Immunol. 58:52-59.

74. Wojcik, W.J., Swoveland, P., Zhang, X., and Vanguri, P. 1996. Chronic intrathecal infusion of phosphorothioate or phosphodiester antisense oligonucleotides against cytokine responsive gene-2/IP-10 in experimental allergic encephalomyelitis of Lewis rat. J. Pharmacol. Exp. Ther. 278:404-410.

75. Sriram, S., and Rodriguez, M. 1997. Indictment of the microglia as the villain in multiple sclerosis. Neurology. 48:464-470.

76. Lucchinetti, C.F., Bruck, W., Rodriguez, M., and Lassmann, H. 1996. Distinct patterns of multiple sclerosis pathology indicates heterogeneity in pathogenesis. Brain Pathol. 6:259-274.

77. Rottman, J.B., et al. 1997. Cellular localization of the chemokine receptor CCR5. Correlation to cellular targets of HIV-1 infection. Am. J. Pathol. 151:1341-1351.

78. Zhou, Y., et al. 1998. Impaired macrophage function and enhanced T celldependent immune response in mice lacking CCR5, the mouse homologue of the major HIV-1 coreceptor. J. Immunol. 160:4018-4026.

79. Meucci, O., et al. 1998. Chemokines regulate hippocampal neuronal signaling and gp120 neurotoxicity. Proc. Natl. Acad. Sci. USA. 95:14500-14505. 